\title{
BUILDING CHANGE DETECTION FROM LIDAR POINT CLOUD DATA BASED ON CONNECTED COMPONENT ANALYSIS
}

\author{
Mohammad Awrangjeb ${ }^{\mathrm{a}, ~}$, Clive S. Fraser ${ }^{\mathrm{b}}$ and Guojun $\mathrm{Lu}^{\mathrm{a}}$ \\ ${ }^{\text {a }}$ School of Engineering and Information Technology, Federation University - Australia \\ Churchill Vic 3842 Australia - (Mohammad.Awrangjeb, Guojun.Lu)@ federation.edu.au \\ ${ }^{\mathrm{b}} \mathrm{CRC}$ for Spatial Information, Dept. of Infrastructure Engineering, University of Melbourne \\ Parkville Vic 3010, Australia - c.fraser@unimelb.edu.au
}

\section{WG III/4}

KEY WORDS: Building detection, change detection, map update, automation, LIDAR, point cloud data

\begin{abstract}
:
Building data are one of the important data types in a topographic database. Building change detection after a period of time is necessary for many applications, such as identification of informal settlements. Based on the detected changes, the database has to be updated to ensure its usefulness. This paper proposes an improved building detection technique, which is a prerequisite for many building change detection techniques. The improved technique examines the gap between neighbouring buildings in the building mask in order to avoid under segmentation errors. Then, a new building change detection technique from LIDAR point cloud data is proposed. Buildings which are totally new or demolished are directly added to the change detection output. However, for demolished or extended building parts, a connected component analysis algorithm is applied and for each connected component its area, width and height are estimated in order to ascertain if it can be considered as a demolished or new building part. Finally, a graphical user interface (GUI) has been developed to update detected changes to the existing building map. Experimental results show that the improved building detection technique can offer not only higher performance in terms of completeness and correctness, but also a lower number of undersegmentation errors as compared to its original counterpart. The proposed change detection technique produces no omission errors and thus it can be exploited for enhanced automated building information updating within a topographic database. Using the developed GUI, the user can quickly examine each suggested change and indicate his/her decision with a minimum number of mouse clicks.
\end{abstract}

\section{INTRODUCTION}

Automation in building change detection is a key issue in the updating of building information in a topographic database. There is increased need for map revision, without large increases in cost, in order to keep the mapping current, especially in those areas which are subject to dynamic change due to new construction and reconstruction of urban features such as building and roads. An updated topographic map along with the required building and road information can be used for a multitude of purposes, including urban planning, identification of informal settlements, telecommunication planning and analysis of noise and air pollution. The availability of high resolution aerial imagery and LIDAR (Light Detection And Ranging) point cloud data has facilitated increased automation within the updating process.

Automatic building change detection from remote sensing data mainly falls into two categories (Grigillo et al., 2011). Firstly, in the direct approach, data acquired from one type of sensor at two different dates are directly compared to detect changes. Secondly, in the indirect approach, the building information is first detected from a new data set and then compared to that in the existing map. For instance, while (Murakami et al., 1999) detected building changes by simply subtracting DSMs (Digital Surface Models) collected on different dates, (Vosselman et al., 2005) first segmented the normalised DSM in order to detect buildings and then compared them to buildings in the map.

The result of automatic building change detection is subsequently used for updating the existing building map. Since the result may not be $100 \%$ correct, some manual work is still required while

\footnotetext{
${ }^{*}$ Corresponding author.
}

updating the database. (Murakami et al., 1999) suggested that omission error (e.g. missing new and/or demolished buildings and/or building parts) in automatic change detection has to be avoided completely in order to keep human involvement to a minimum. This is because in the worst case scenario a possibility of omission error would require a manual inspection of the entire original data. Thus, as long as no omission errors are included in the result, removal of commission errors (e.g., false identification of new and/or demolished buildings and/or building parts) can be made in a straightforward manner by focusing expensive manual inspection only on detected changes, which can then reduce the time required to investigate the whole area of interest.

In 2009, under a project of the European Spatial Data Research organisation, (Champion et al., 2009) compared four building change detection techniques and stated that only the method of (Rottensteiner, 2007) had achieved a relatively acceptable result. Nevertheless, the best change detection technique in their study was still unsuccessful in reliable detection of small buildings. So, there is significant scope for investigation of new automatic building change detection approaches.

This paper proposes a new method for updating building information in a topographic map using LIDAR point cloud data. Firstly, it presents an improved automatic building detection technique that reduces instances of under segmentation in densely builtup areas. Then, it proposes a new automatic building change detection approach by comparing the extracted building information with that in an existing building database. A connecting component analysis-based technique is proposed to remove the false changes and thereby identify actual changes. Finally, a simple user interface (GUI) is developed to allow the user to quickly rectify the building change detection result before the 
map database is actually updated. The GUI can be facilitated with an orhtophoto of the study area.

\section{RELATED WORK}

For both building detection and change detection, there are three categories of technique, based on the input data: image only (Ioannidis et al., 2009, Champion et al., 2010), LIDAR only (Vosselman et al., 2005) and the combination of image and LIDAR (Rottensteiner, 2007). The comprehensive comparative study of (Champion et al., 2009) on building change detection techniques showed that LIDAR-based techniques offer high economic effectiveness. Thus, this paper concentrates on techniques that use at least LIDAR data.

Amongst reported building detection approaches, (Rottensteiner, 2007) employed a classification using the Dempster-Shafer theory of data fusion to detect buildings from LIDAR derived DSMs and multi-spectral imagery. For building detection from LIDAR point cloud data alone, (Zhang et al., 2006) first separated the non-ground points from the ground points using a progressive morphological filter. The buildings were then separated from trees using non-ground points by applying a region-growing algorithm based on a plane-fitting technique. (Awrangjeb and Fraser, 2014b) simply applied a height threshold to separate the ground and non-ground points. A region growing technique was then applied to grow planes on the stable seed points. A set of rules were applied to remove planes constructed on trees. Finally, neighbouring planes were combined to form individual building boundaries. Reviews of other building detection techniques can be found in (Zhang et al., 2006, Awrangjeb and Fraser, 2014b).

In regard to building change detection, (Zong et al., 2013) proposed a direct change detection technique by fusing high-resolution aerial imagery with LIDAR data through a hierarchical machine learning framework. After initial change detection, a post processing step based on homogeneity and shadow information from the aerial imagery, along with size and shape information of buildings, were applied to refine the detected changes. Promising results were obtained in eight small test areas. (Murakami et al., 1999) subtracted one of the LIDAR DSMs from three others acquired on four different dates. The difference images were then rectified using a straightforward shrinking and expansion filter that reduced the commission errors. The parameter of the filter was set based on prior knowledge about the horizontal error in the input LIDAR data. The authors reported no omission error for the test scene, but they did not provide any statistics on the other types of measurements.

Among the indirect approaches to building change detection, (Matikainen et al., 2010) used a set of thresholds to determine building changes and showed that the change detection step is directly affected by the preceding building detection step, particularly when buildings have been missed due to low height and tree coverage. Thus, a set of correction rules based on the existing building map was proposed and the method was tested on a large area. High accuracy was achieved (completeness and correctness were around $85 \%$ ) for buildings larger than $60 \mathrm{~m}^{2}$, however the method failed to detect changes for small buildings.

(Grigillo et al., 2011) used the exclusive-OR operator between the existing building map and the newly detected buildings to obtain a mask of building change. A set of overlap thresholds (similar to (Matikainen et al., 2010)) to identify demolished, new, extended and discovered old (unchanged) buildings was then applied and a per-building completeness and correctness of $93.5 \%$ and $78.4 \%$, respectively, was achieved. (Rottensteiner, 2007) compared two

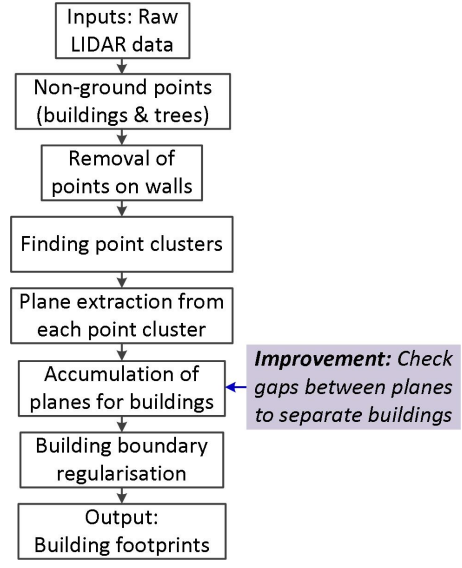

Figure 1: The proposed building detection technique.

label images (from the existing map and automatic building detection) to decide building changes (confirmed, changed, new and demolished). Although, high per-pixel completeness and correctness measures (95\% and $97.9 \%$ ) were obtained, the method missed small structures. The method by (Vosselman et al., 2005) ruled out the buildings not visible from the roadside (e.g., buildings in the back yard) and also produced some mapping errors.

\section{PROPOSED IMPROVED BUILDING DETECTION TECHNIQUE}

Fig. 1 shows the work flow of a previously developed building detection technique (Awrangjeb and Fraser, 2014b), with the proposed improvement to be presented here being highlighted. This technique first divides the input LIDAR point cloud data into ground and non-ground points. The non-ground points, representing objects above the ground such as buildings and trees, are further processed for building extraction. Points on walls are removed from the non-ground points, which are then divided into clusters. Planar roof segments are extracted from each cluster of points using a region-growing technique. Planar segments constructed in trees are eliminated using information such as area, orientation and unused LIDAR points within the plane boundary. Points on the neighbouring planar segments are accumulated to form individual building regions. An algorithm is employed to regularise the building boundary. The final output from the method consists of individual building footprints.

Application of this building detection technique has shown that it can offer a high number of under-segmentation results when buildings are close to each other, especially in low density point cloud data. For example, Fig. 2a shows the input (non-ground) point cloud for three neighbouring buildings. Although, three buildings are clearly separable from each other, the building detection technique detects a merged building as shown in Fig. $2 \mathrm{~b}$. This is because when the segmented neighbouring roof planes are accumulated to form individual buildings, a distance threshold $T_{d}$ is used to find the neighbouring roof planes. The value of $T_{d}$ is set to twice the maximum point spacing in the input point cloud data. Thus, for low density input data, when the value of $T_{d}$ is quite high, the segmented planes from nearby building roofs are accumulated to form a single building, although the buildings are found well-separated in the building mask (Fig. 2c).

In order to avoid such an expected merging, the white gap between two buildings in the building mask, shown in Fig. 2d, is investigated in the proposed improvement. For two real neighbouring planes on a building, there should be no white gap, i.e. 


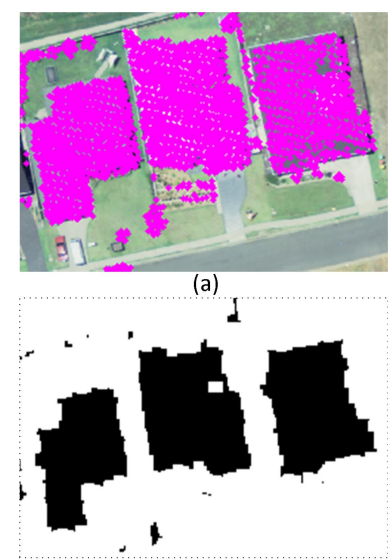

(c)

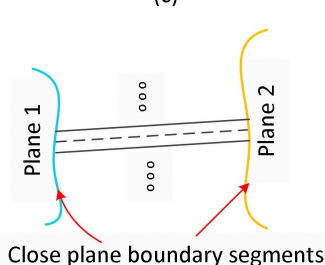

(e)

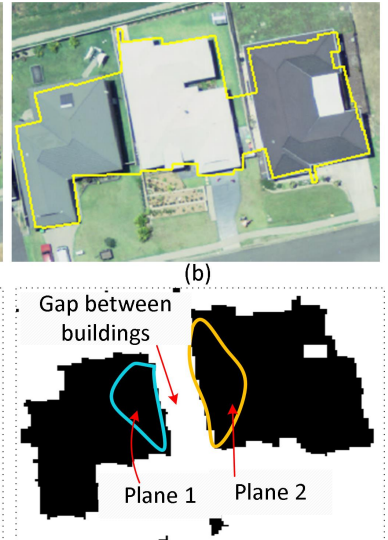

(d)

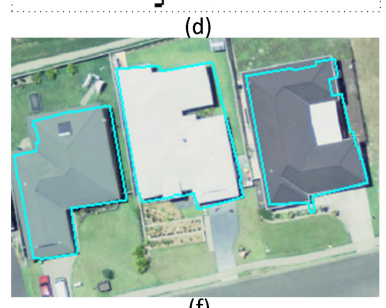

(f)

Figure 2: Separating nearby buildings: (a) input (non-ground) point cloud data, (b) extracted building footprint (buildings are merged), (c) corresponding building mask, (d) boundaries of two neighbouring roof planes, (e) close segments of plane boundaries (dashed line to test gap between planes) and (f) improved building footprints (buildings are separated).

they should be connected by continuous black pixels in the building mask. In contrast, for two planes from well-separated neighbouring buildings there should be a white gap between the planes in the building mask. Thus, after choosing two neighbouring roof planes based on $T_{d}$ (see Fig. 2d), the following test is carried out before considering them to be from the same building. First, the close segments of the two plane boundaries (within $T_{d}$ ) are identified, as shown in Fig. 2e. Then, a line is connected between boundary segments by connecting their two midpoints (dashed line in Fig. 2e). By generating sample points on the line, it can be easily tested whether the line passes through only the black pixels of the mask. If it does, then the two neighbouring planes are deemed to be from the same building. If it does not, then more lines are formed by connecting points on the boundary segments (solid lines in Fig. 2e) and they are iteratively tested. If none of the lines passes through only black pixels, then the two planes are identified as being from different buildings.

The above test is effective if the gap between the two buildings is large enough such that LIDAR points can hit the ground in between the buildings so as to create a white gap in the building mask. If the point density is low and there are no LIDAR returns from the ground between the buildings, the building detection technique may still find the neighbouring buildings to be merged.

\section{PROPOSED BUILDING CHANGE DETECTION TECHNIQUE}

The proposed building change detection technique is presented here with the help of Fig. 3. The technique uses two sets of building information as input: an existing building map and the automatically detected building footprints from a new data set (Figs. 3a-b). In order to obtain changes between the inputs, four masks of resolution 0.5 or $1 \mathrm{~m}$ (e.g., resolution of the inputs) are generated. The first mask $M_{1}$, shown in Fig. 3c, is a coloured

mask that shows three types of building regions. The blue regions indicate no change, i.e. buildings exist in both the inputs. The red regions indicate building regions that exist only in the existing map. The green regions indicate new building regions, i.e. the newly detected building outlines.

The second mask $M_{2}$, depicted in Fig. 3d, is a binary mask that contains only the old building parts (not the whole buildings). As shown in Fig. 3g, the third mask $M_{3}$ is also a binary mask that contains only the new building parts (again, not the whole buildings).

The fourth mask $M_{4}$ is a coloured mask that shows the final building change detection results (see Fig. 3d). Initially, the new and old (demolished) 'whole building' regions are directly transferred from $M_{1}$. Then, demolished and new building parts are marked in the final mask after the following assessment is applied to $M_{2}$ and $M_{3}$

There may be misalignment between the buildings from the two input data sources. As a result, there can be many unnecessary edges and thin black regions found in $M_{2}$ and $M_{3}$. These small errors in either mask increase the chance that buildings will be incorrectly classified as changed. Assuming that the minimum width of an extended or demolished building part is $W_{m}=3 \mathrm{~m}$ (Vosselman et al., 2005), a morphological opening filter with a square structural element of $3 \mathrm{~m}$ is applied to $M_{2}$ and $M_{3}$ separately. As can be seen in Figs. $3 \mathrm{e}$ and h, the filtered masks $M_{2 f}$ and $M_{3 f}$ are now almost free of misalignment problems.

Next, a connected component analysis algorithm is applied to $M_{2 f}$ and $M_{3 f}$ separately. The algorithm returns an individual connected component along with its area (number of pixels), centroid and the orientation of the major axis of the ellipse that has the same normalized second central moments as the component's region. Small regions (areas less than $9 \mathrm{~m}^{2}$ ) are removed.

Fig. 3f shows a region from $M_{3 f}$ in Fig. 3h, along with its centroid $C$ and the ellipse with its two axes. The width and length of the region can now be estimated by counting the number of black pixels along the two axes that pass through $C$. If both the length and width are at least $W_{m}$ then the region is accepted as a demolished (for $M_{2 f}$ ) or new (extended, for $M_{3 f}$ ) building part. If they are not, $C$ moves along/across the major and/or minor axes to iteratively check if the region has the minimum required size. In Fig. 3i, a new position of $C$ is shown as $C^{\prime}$ and the two lines parallel to the two axes are shown by dashed lines.

The demolished and extended building parts are consequently marked (using pink and yellow colours, respectively) in $M_{4}$, which is the final output of the proposed change detection technique. Although there are many under-segmentation cases (i.e. buildings are merged as seen in Fig. 3b), Fig. 3i shows that the proposed change detection technique is robust against such segmentation errors being propagated from the building detection phase. Moreover, it is free from omission errors (i.e., failure to identify any real building changes), but it has some commission errors, such as trees coming from the building detection step, as shown by the yellow regions in Fig. 3i.

\section{UPDATING THE BUILDING MAP}

A simple GUI (graphical user interface) has been developed to allow the user to update the existing building map using the automated change detection results. As shown in Fig. 4, the GUI shows the existing building outlines using black polygons and the final change result (Mask $M_{4}$ ) using dots in different colours. 

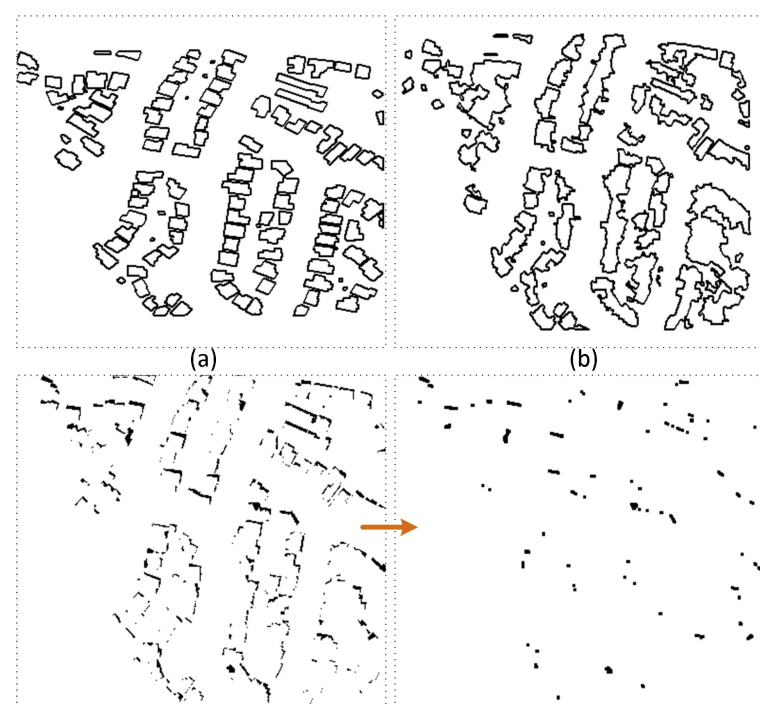

(d)

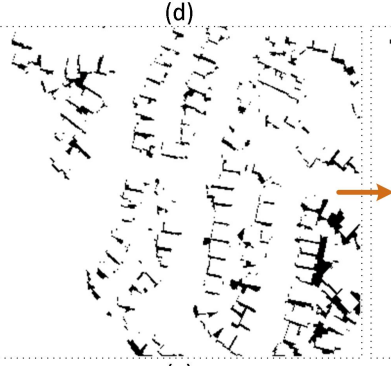

(g)

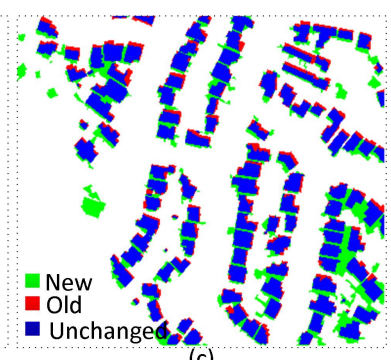

(c)

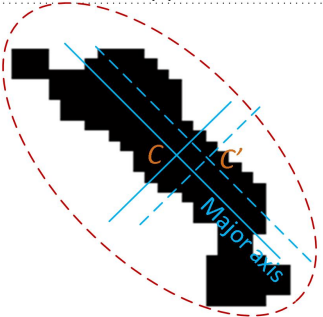

(e) (f)

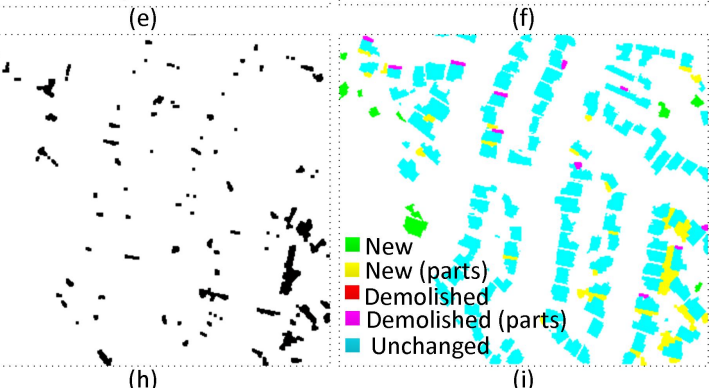

(h)

Figure 3: Change detection: (a) existing building map, (b) extracted buildings from new data, (c) mask from (a) \& (b) showing old, new and existing building/parts, (d) mask for old building parts only, (e) morphological opening of (d), (f) estimation of length and width of a component in (e) or (h), (g) mask for new building parts only, (h) morphological opening of (g), (i) final change detection results.

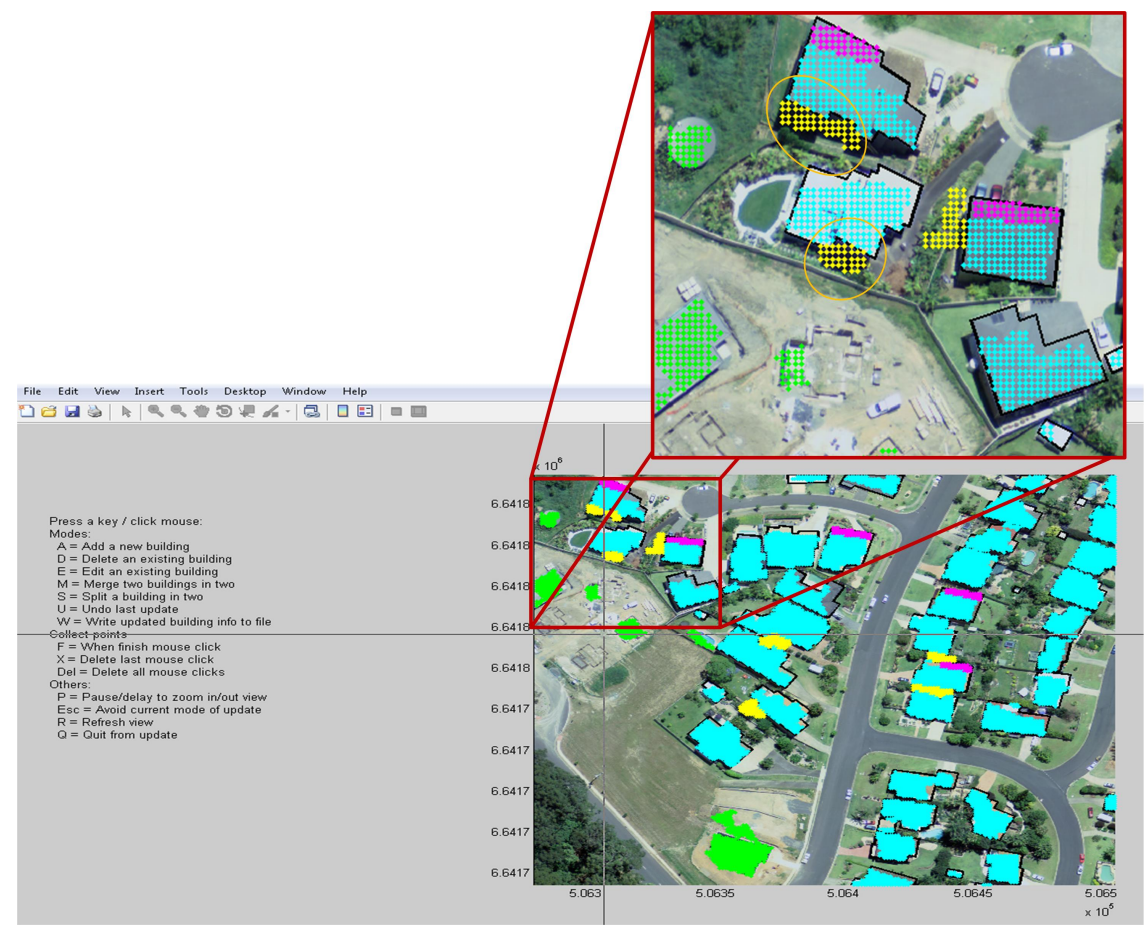

Figure 4: A simple graphical user interface (GUI) for user to update the building map.

Thus, the user can look at the suggested colour-coded changes overlaid on the orthophoto and decide which should be applied to the map (see magnified snapshot in Fig. 4). The user can simply avoid a commission error by taking no action, and an actual change can be accepted by selecting one or two of the functional tools in the GUI, which in general has the following functions:

1. Addition: Looking at a green region, which is a suggested true new building, the user can click a mouse button on the 
region. The corresponding green region will then be added as a building footprint. Alternatively, the user can draw a regular polygon for the new building.

2. Deletion: In order to delete a true demolished building the user can either click on the suggested red region or can draw a polygon around one or more demolished buildings to specify the region. To avoid a suggested addition (green or yellow regions), the user can simply click on the region.

3. Merge: In order to merge a true new building part (yellow region), the user first adds the part to the map using the addition tool above and then merges the addition with an existing building by clicking on the two boundary segments to be merged. Alternatively, the changed building in the existing map can be directly replaced with its extended version from the building detection phase.

4. Split: In order to delete a true demolished building part (pink region), the user clicks on the pink region, which will be removed from the corresponding existing building. Or, the user can click on consecutive points along which a building must be split. The part that contains the yellow region will be removed. Alternatively, the changed building in the existing map can be directly replaced with its new version from the building detection phase.

5. Edit: The user can rectify any other building delineations in the existing map. This will be helpful when a recent highresolution orthophoto is available.

6. Undo: The user can undo the previous action.

7. Save: The updated map can be saved at any time

In general, the GUI is capable of generating a new building map by simply using an orthophoto and/or automatically extracted building footprints.

\section{STUDY AREA}

In order to validate the proposed building change detection technique, LIDAR point cloud data from two different dates (September and December 2009) have been employed. The data sets covered the area of Coffs Harbour in NSW, Australia. Four test scenes were selected from the whole area based on the change in buildings. Due to the short time interval of three months, newly constructed buildings were the main factor in selecting the areas. All the test areas were moderately vegetated.

There were also orthophotos available for the test areas. The image resolution was $50 \mathrm{~cm}$ and $10 \mathrm{~cm}$ for two captured dates in September and December, respectively. These orthophotos were mainly used to manually collect the reference building information. They were also used in the GUI to make decisions on the result from the automated change detection technique.

Table 1 shows some characteristics of the test scenes for both of the dates. While the point density (number of points divided by the area) was similar at 0.35 points $/ \mathrm{m}^{2}$ for all scenes in September, it varied in December. There were no demolished buildings and the number of new buildings in the four scenes was 18,6 , 3 and 1, respectively. In Scenes 2 and 3 there were buildings smaller than $10 \mathrm{~m}^{2}$ and $25 \mathrm{~m}^{2}$ in area. In Scene 2, there were also some new buildings which were smaller than $25 \mathrm{~m}^{2}$ and 50 $\mathrm{m}^{2}$ in area. There were only two demolished building parts in Scenes $1 \& 3$, but no new building parts.

\begin{tabular}{|l|cccc|}
\hline Scenes & Dimension & P density & Buildings & B size \\
\hline \hline 1, Sep & $343 \times 191$ & 0.36 & 17 & $0,0,0$ \\
1, Dec & $343 \times 191$ & 2.28 & 35 & $0,0,1$ \\
\hline 2, Sep & $378 \times 318$ & 0.35 & 117 & $1,9,11$ \\
2, Dec & $378 \times 318$ & 1.43 & 123 & $1,11,17$ \\
\hline 3, Sep & $166 \times 254$ & 0.37 & 41 & $1,2,7$ \\
3, Dec & $166 \times 254$ & 3.02 & 44 & $1,2,7$ \\
\hline 4, Sep & $149 \times 150$ & 0.35 & 1 & $0,0,0$ \\
4, Dec & $149 \times 150$ & 1.26 & 2 & $0,0,0$ \\
\hline \hline
\end{tabular}

Table 1: Four test data sets, each from two dates: dimensions in metres, point density in points $/ \mathrm{m}^{2}, \mathrm{~B}$ size indicates the number of buildings within $10 \mathrm{~m}^{2}, 25 \mathrm{~m}^{2}$ and $50 \mathrm{~m}^{2}$ in area, respectively.

\begin{tabular}{|c|c|c|c|c|c|c|c|}
\hline$C_{m}$ & $C_{r}$ & $C_{m, 10}$ & $C_{r, 10}$ & $C_{m, 50}$ & $C_{r, 50}$ & $N_{m}$ & $N_{s}$ \\
\hline \hline \multicolumn{7}{|c|}{ Scene 1 (first row shows improved results) } \\
\hline 100 & 100 & 100 & 100 & 100 & 100 & 0 & 0 \\
100 & 100 & 100 & 100 & 100 & 100 & 0 & 0 \\
\hline \multicolumn{7}{|c|}{ Scene 2 (first row shows improved results) } \\
\hline 84.9 & 94.8 & 85.9 & 94.8 & 96.1 & 94.8 & 12 & 22 \\
82.4 & 94.9 & 83.6 & 94.9 & 96.6 & 94.9 & 10 & 32 \\
\hline \multicolumn{7}{|c|}{ Scene 3 (first row shows improved results) } \\
\hline 83.9 & 96.3 & 83.9 & 96.3 & 96.3 & 96.3 & 0 & 5 \\
81.5 & 95.7 & 81.5 & 95.7 & 95.7 & 95.7 & 0 & 8 \\
\hline \multicolumn{7}{|c|}{ Scene 4 (first row shows improved results) } \\
\hline 100 & 100 & 100 & 100 & 100 & 100 & 0 & 0 \\
100 & 100 & 100 & 100 & 100 & 100 & 0 & 0 \\
\hline \multicolumn{7}{|c|}{ Average on 4 scenes (first row shows improved results) } \\
\hline 92.2 & 97.8 & 92.5 & 97.8 & 98.1 & 97.8 & 3.0 & 6.75 \\
91.0 & 97.7 & 91.3 & 97.7 & 98.1 & 97.6 & 2.5 & 10.0 \\
\hline
\end{tabular}

Table 2: Comparing object-based building detection results in September data sets with and without the application of the proposed improvement. $C_{m}=$ completeness and $C_{r}=$ correctness $\left(C_{m, 10}, C_{r, 10}\right.$ and $C_{m, 50}, C_{r, 50}$ are for buildings over $10 \mathrm{~m}^{2}$ and $50 \mathrm{~m}^{2}$, respectively) are in percentage. $N_{s}=$ number of split operations and $N_{m}=$ number of merge operations.

\section{EXPERIMENTAL RESULTS}

The experimental results are presented for building detection, change detection and map update separately.

\subsection{Building detection results}

The performance of the building detection step was evaluated using the object-based completeness and correctness measures, as well as the number of split and merge operations (Awrangjeb and Fraser, 2014a). Split operations are required during evaluation due to under-segmentation errors when two or more buildings are detected as one single building. Conversely, merge operations are required due to over-segmentation errors when a single building is detected as two or more buildings.

Table 2 compares the evaluation results on the September data sets, with and without the application of the proposed improvement presented in Section 3. Table 3 shows the same for the December data sets.

The proposed improvement reduced the number of split operations considerably, especially in Scenes 2 and 3 in September and Scenes 1, 2 and 3 in December. As can be seen in Fig. 5, the proposed improvement was effective in identifying individual building boundaries, which reduced the number of required split operations during the evaluation. However, the improvement moderately increased the over-segmentation error, especially in 


\begin{tabular}{|c|c|c|c|c|c|c|c|}
\hline$C_{m}$ & $C_{r}$ & $C_{m, 10}$ & $C_{r, 10}$ & $C_{m, 50}$ & $C_{r, 50}$ & $N_{m}$ & $N_{s}$ \\
\hline \hline \multicolumn{7}{|c|}{ Scene 1 (first row shows improved results) } \\
\hline 100 & 86.1 & 100 & 86.1 & 100 & 86.1 & 5 & 3 \\
100 & 81 & 100 & 81 & 100 & 81 & 2 & 12 \\
\hline \multicolumn{7}{|c|}{ Scene 2 (first row shows improved results) } \\
\hline 92.5 & 81.7 & 94.2 & 81.7 & 100 & 81.7 & 14 & 60 \\
82.4 & 60.9 & 87.5 & 60.9 & 100 & 60.9 & 0 & 84 \\
\hline \multicolumn{7}{|c|}{ Scene 3 (first row shows improved results) } \\
\hline 95.2 & 83.3 & 95.2 & 83.3 & 100 & 83.3 & 3 & 15 \\
100 & 66.7 & 100 & 66.7 & 100 & 66.7 & 0 & 34 \\
\hline \multicolumn{7}{|c|}{ Scene 4 (first row shows improved results) } \\
\hline 100 & 100 & 100 & 100 & 100 & 100 & 0 & 0 \\
100 & 100 & 100 & 100 & 100 & 100 & 0 & 0 \\
\hline \multicolumn{7}{|c|}{ Average on 4 scenes (first row shows improved results) } \\
\hline 96.9 & 87.8 & 97.4 & 87.8 & 100 & 87.8 & 5.5 & 19.5 \\
95.6 & 77.2 & 96.9 & 77.2 & 100 & 77.2 & 0.5 & 32.5 \\
\hline \hline
\end{tabular}

Table 3: Comparing object-based building detection results in December data sets with and without the application of the proposed improvement. $C_{m}=$ completeness and $C_{r}=$ correctness $\left(C_{m, 10}, C_{r, 10}\right.$ and $C_{m, 50}, C_{r, 50}$ are for buildings over $10 \mathrm{~m}^{2}$ and $50 \mathrm{~m}^{2}$, respectively) are in percentage. $N_{s}=$ number of split operations and $N_{m}=$ number of merge operations.

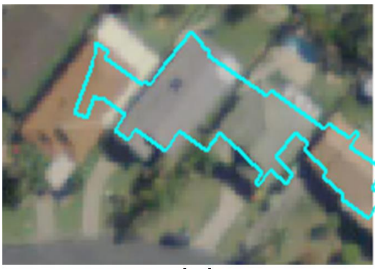

(a)

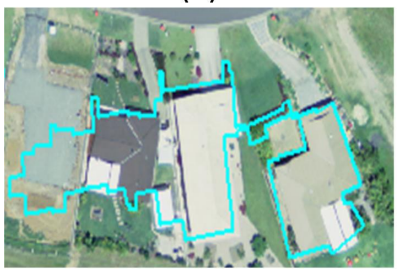

(c)

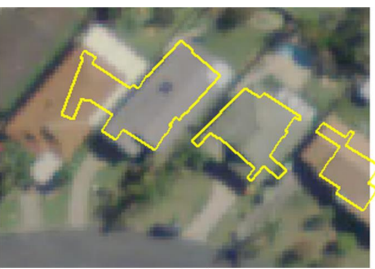

(b)

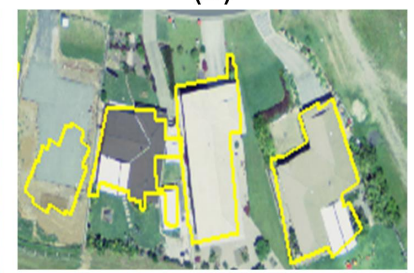

(d)

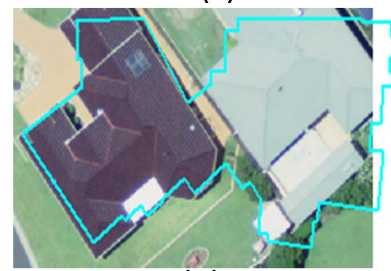

(e)

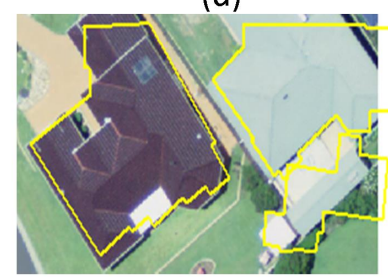

(f)

\begin{tabular}{|l|cc|cccc|cccc|}
\hline & \multicolumn{3}{|c|}{ Reference } & \multicolumn{4}{|c|}{ Change det. } & \multicolumn{4}{c|}{ Comm. error } \\
\hline Scenes & N & DP & N & D & NP & DP & N & D & NP & DP \\
\hline \hline 1 & 18 & 1 & 27 & 1 & 1 & 1 & 9 & 1 & 1 & 0 \\
2 & 6 & 0 & 18 & 2 & 97 & 66 & 12 & 2 & 97 & 66 \\
3 & 3 & 1 & 8 & 0 & 15 & 1 & 5 & 0 & 15 & 0 \\
4 & 1 & 0 & 1 & 0 & 0 & 0 & 0 & 0 & 0 & 0 \\
\hline \hline
\end{tabular}

Table 4: Change detection results with removal of commission errors $>9 \mathbf{m}^{2}$ in area. $\mathrm{N}=$ new buildings, $\mathrm{D}=$ demolished buildings, $\mathrm{NP}=$ new building parts and $\mathrm{DP}=$ demolished building parts.

\begin{tabular}{|l|cc|cccc|cccc|}
\hline & \multicolumn{3}{|c|}{ Reference } & \multicolumn{4}{|c|}{ Change det. } & \multicolumn{4}{c|}{ Comm. error } \\
\hline Scenes & N & DP & N & D & NP & DP & N & D & NP & DP \\
\hline \hline 1 & 18 & 1 & 20 & 1 & 1 & 1 & 2 & 1 & 1 & 0 \\
2 & 6 & 0 & 10 & 0 & 27 & 11 & 4 & 0 & 27 & 11 \\
3 & 3 & 1 & 6 & 0 & 13 & 1 & 3 & 0 & 13 & 0 \\
4 & 1 & 0 & 1 & 0 & 0 & 0 & 0 & 0 & 0 & 0 \\
\hline \hline
\end{tabular}

Table 5: Change detection results with removal of commission errors $>25 \mathrm{~m}^{2}$ in area. $\mathrm{N}=$ new buildings, $\mathrm{D}=$ demolished buildings, $\mathrm{NP}=$ new building parts and $\mathrm{DP}=$ demolished building parts.

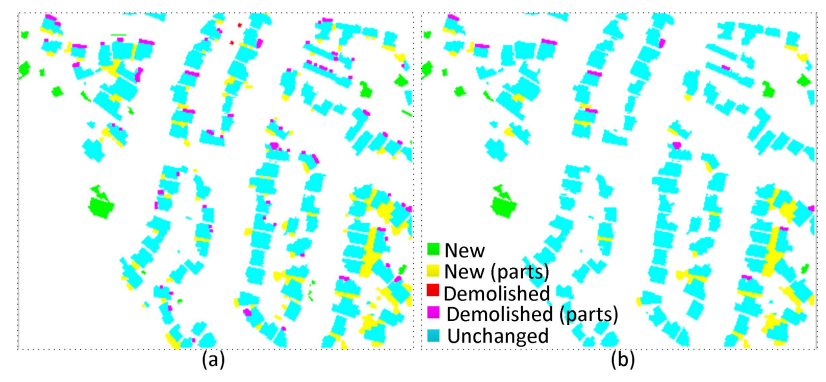

Figure 6: Building change detection in Scene 2: larger than (a) 9 $\mathrm{m}^{2}$ and (b) $25 \mathrm{~m}^{2}$ (small regions removed).

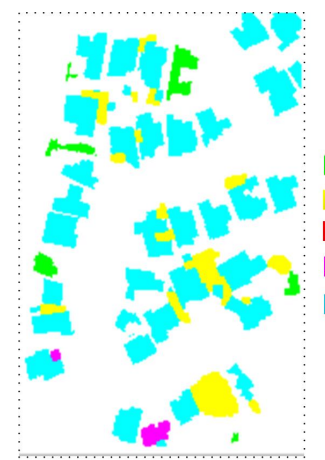

(a)

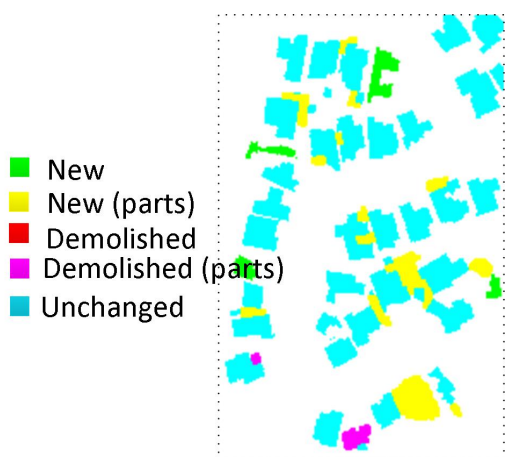

(b)

Figure 5: Building detection examples: with (left) and without (right) the improvement in Section 3. Row 1 from Scene 2 and Rows 2 \& 3 from Scene 3.

the December data sets where connected building parts were separated (see the example in Fig. 5f, originally together in the reference data set).

Comparing the results from September and December, high completeness values were observed in December due to higher point density, which allowed detection of some small buildings in both Scenes $2 \& 3$. However, high point density also allowed some detection of trees, which reduced the correctness. This might also be the case because the vegetation became denser during the Spring (Sep-Dec). The number of split operations was lower in September than in December, because low point density data kept the segmented planes from neighbouring buildings further away

Figure 7: Building change detection in Scene 3: larger than (a) 9 $\mathrm{m}^{2}$ and (b) $25 \mathrm{~m}^{2}$ (small regions removed).

from each other.

\subsection{Change detection results}

Tables 4 and 5 show the change detection results for the test scenes. Although, there were no omission errors (missing new and/or demolished buildings and/or building parts), there were a significant number of commission errors (false identification of new and/or demolished buildings and/or building parts), mainly propagated from the building detection step, especially in Scenes $2 \& 3$.

False new buildings and building parts comprised trees that the building detection step could not remove. Some buildings were 


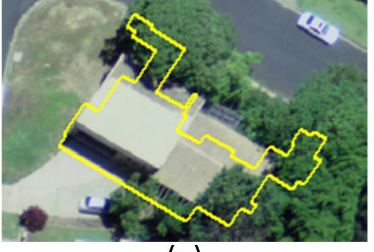

(a)

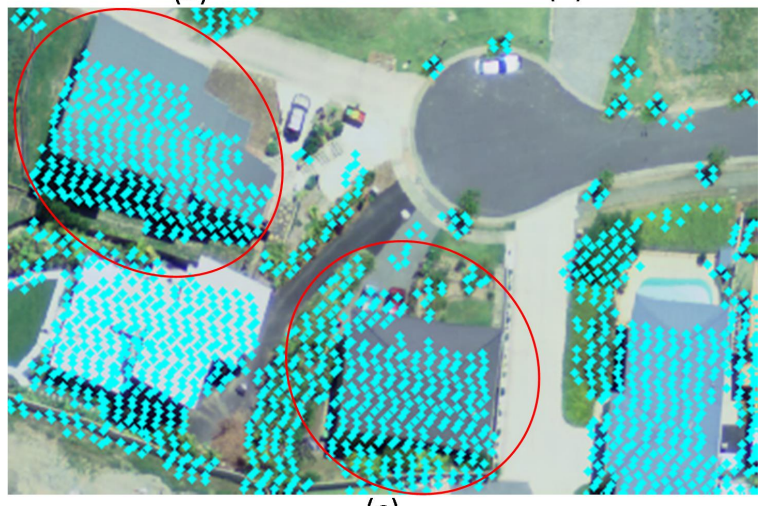

(c)

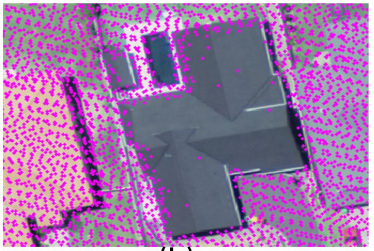

(b)

Figure 8: Some difficulties in building detection: (a) extended over the occluding tree, (b) missing building due to absence of point cloud data and (c) registration error between orthoimage and point cloud data in Scene 2.

found to be extended over neighbouring trees (see Fig. 8a) and some trees were detected as separate buildings. There was one false demolished building due to the absence of data in the input point cloud (see Fig. 8b). Also, there were many false demolished build parts. This exclusively happened in Scene 2, as shown in Fig. 6a, and was due to registration error between the existing building map, which was manually created from the supplied orthophoto, and the automatically detected building footprints from the LIDAR point cloud. An example of this registration error is shown within the red circles in Fig. 8c. The registration error also led to some false detection of extended buildings, as shown within orange circles in the magnified version of Fig. 4. However, in the other three scenes the number of false demolished building parts was low because the registration error was negligible. For example, Fig. 7 shows the change detection result for Scene 3.

\begin{tabular}{|l|ccccccc|}
\hline Scenes & D & A & S & M & NB & EB & NM \\
\hline 1, Sep & 0 & 0 & 0 & 0 & 2 & 1.5 & 7.3 \\
2, Sep & 3 & 12 & 14 & 5 & 58 & 1.3 & 4.8 \\
3, Sep & 1 & 5 & 4 & 0 & 15 & 1.5 & 4.6 \\
4, Sep & 0 & 0 & 0 & 0 & 1 & 2 & 3 \\
\hline Avg & $\mathbf{1}$ & $\mathbf{4 . 3}$ & $\mathbf{4 . 5}$ & $\mathbf{1 . 3}$ & $\mathbf{1 9}$ & $\mathbf{1 . 6}$ & $\mathbf{4 . 9}$ \\
\hline 1, Dec & 7 & 1 & 2 & 3 & 12 & 1.5 & 4.3 \\
2, Dec & 8 & 4 & 46 & 1 & 55 & 1.3 & 3.6 \\
3, Dec & 3 & 1 & 11 & 0 & 18 & 1.5 & 4.6 \\
4, Dec & 0 & 0 & 0 & 0 & 1 & 1 & 2 \\
\hline Avg & $\mathbf{4 . 5}$ & $\mathbf{1 . 5}$ & $\mathbf{1 4 . 8}$ & $\mathbf{1}$ & $\mathbf{2 1 . 5}$ & $\mathbf{1 . 3}$ & $\mathbf{3 . 6}$ \\
\hline
\end{tabular}

Table 6: Estimation of manual interactions to generate map using automatically extracted buildings. $\mathrm{D}=$ number of deletions for removing false buildings (trees), $\mathrm{A}=$ number of additions for inclusion of missing true buildings, $S=$ number of split operations, $\mathrm{M}=$ number of merge operations, $\mathrm{NB}=$ number of detected buildings being edited to be acceptable, $\mathrm{EB}=$ number of editions per edited building and $\mathrm{NM}=$ number of mouse clicks per edition.

\subsection{Map from automatic buildings vs point density}

The simple GUI was mainly used for user interaction to quickly accept or reject the suggested changes. Fig. 9a shows the updated

\begin{tabular}{|l|c|c|c|c|c|c|}
\hline Scenes & $C_{m}$ & $C_{r}$ & $Q_{l}$ & $A_{o}$ & $A_{c}$ & $R M S E$ \\
\hline 1 & 64.1 & 90.2 & 59.9 & 35.9 & 9.8 & 2.8 \\
2 & 79.0 & 86.3 & 70.1 & 22.0 & 14.5 & 2.1 \\
3 & 65.0 & 77.6 & 54.7 & 39.0 & 25.2 & 2.9 \\
4 & 85.0 & 98.8 & 84.0 & 15.1 & 1.3 & 1.1 \\
\hline Avg & $\mathbf{7 3 . 2}$ & $\mathbf{8 8 . 2}$ & $\mathbf{6 7 . 2}$ & $\mathbf{2 8 . 0}$ & $\mathbf{1 2 . 7}$ & $\mathbf{2 . 2}$ \\
\hline
\end{tabular}

Table 7: Pixel-based evaluation results for September data set for the generated map from the automatically extracted buildings. $C_{m}=$ completeness, $C_{r}=$ correctness, $Q_{l}=$ quality, $A_{o}=$ area omission error and $A_{c}=$ area commission error are in percentage. $R M S E=$ root mean square error in metre.

\begin{tabular}{|l|c|c|c|c|c|c|}
\hline Scenes & $C_{m}$ & $C_{r}$ & $Q_{l}$ & $A_{o}$ & $A_{c}$ & $R M S E$ \\
\hline 1 & 92.3 & 88.3 & 82.3 & 7.7 & 11.7 & 1.8 \\
2 & 81.5 & 81.8 & 68.9 & 19.5 & 19.1 & 2.1 \\
3 & 87.2 & 87.5 & 77.6 & 13.2 & 12.8 & 2.0 \\
4 & 93.2 & 93.2 & 87.2 & 6.8 & 6.8 & 1.6 \\
\hline Avg & $\mathbf{8 8 . 5}$ & $\mathbf{8 7 . 7}$ & $\mathbf{7 9 . 0}$ & $\mathbf{1 1 . 8}$ & $\mathbf{1 2 . 6}$ & $\mathbf{1 . 9}$ \\
\hline
\end{tabular}

Table 8: Pixel-based evaluation results for December data set for the generated map from the automatically extracted buildings. $C_{m}=$ completeness, $C_{r}=$ correctness, $Q_{l}=$ quality, $A_{o}=$ area omission error and $A_{c}=$ area commission error are in percentage. $R M S E=$ root mean square error in metre.

map for Scene 1.

In the case where a building map is not available for an area, the GUI can also be used to generate a map from the automatically extracted building footprints for that area. Thus, for all eight data sets (4 scenes, each from two dates) new building maps were generated from their extracted footprints via the GUI, with minimum user interaction. False buildings were simply deleted and missing buildings were added. There were also merge and split operations to handle over- and under-segmentation cases. As a final step, a number of edits were required for many buildings to make a visually acceptable building map. Figs. 9b-c show an example for Scene 2, December.

Table 6 shows an estimation of the manual works that was 'minimally' required for the eight data sets. It is evident that the December data required more deletions and more split operations than for the September data, as many trees were detected and many neighbouring buildings were merged. Nevertheless, the December data required less additions since many missing buildings had been automatically detected because of the higher point density. The four December data sets required less editing and less mouse clicks per edit. This indicates, as could be anticipated, that detected buildings from a higher density point cloud data contain more detailed information and so require less user interaction.

Table 7 and 8 show pixel-based accuracy for the generated maps (with respect to the manually generated reference buildings). Although the maps from two dates showed similar average correctness and commission errors, on average the December maps provided higher completeness and quality and lower omission and geometric errors. Again, better performance is evident with higher density point cloud data.

\section{CONCLUSION}

This paper has presented a new method for both building change detection and the subsequent update of building changes in a topographic database. Firstly, an improved building detection technique has been proposed that investigates the gap between neigh- 


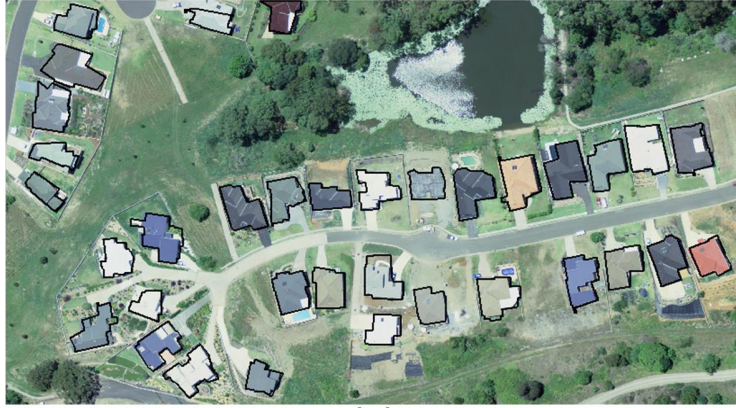

(a)

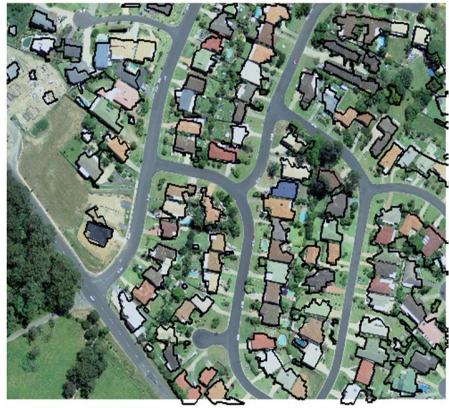

(b)

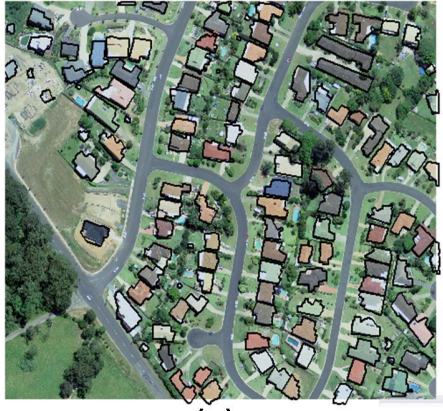

(c)

Figure 9: Map from the extracted buildings: (a) updated map for Scene 1, (b) automatic building detection for Scene 2 in December and (c) map from (b) using the GUI.

bouring buildings in the building mask to avoid any wrong merging of neighbouring buildings. When compared to the original building detection technique, the improved technique offers not only higher performance in terms of completeness and correctness, but also a lower number under-segmentation errors, with a small increase in over-segmentation errors.

An automatic building change detection technique based on connected component analysis has also been presented. Buildings which are totally new or demolished are directly added to the change detection output. However, for demolished or extended building parts, a connected component analysis algorithm is applied, and for each connected component its area, width and height are estimated in order to ascertain if it can be considered as a demolished or extended new building part. Experimental results have shown that the proposed change detection technique provides no omission errors, and thus it can be cheaply exploited for building outline map updating. The resulting high number of commission errors was mainly due to two reasons. Firstly, the building detection technique could not remove all trees and it merged some occluded nearby trees with the detected true buildings. The high registration error between the exiting map (generated from the orthophotos) also resulted in many false new/demolis building parts. Nonetheless, the commission error does impede the required user interaction, since the user only needs to look at the indicated changes and to accept or reject them. Moreover, this high false change detection of small (new/demolished) building parts is an indication that the proposed detection technique is capable of detecting such real changes in an appropriate data set.

Future research includes reconstruction of building roofs and three dimensional change detection of buildings. While both the point cloud data and high resolution aerial imagery can be used for roof modelling, 3D planes from the point cloud data can be exploited for $3 \mathrm{D}$ change detection.

\section{ACKNOWLEDGMENT}

Dr. Awrangjeb is the recipient of the Discovery Early Career Researcher Award by the Australian Research Council (project number DE120101778). The Coffs Harbour data set was provided by Land and Property Information of the NSW Government (www.lpi.nsw.gov.au).

\section{REFERENCES}

Awrangjeb, M. and Fraser, C. S., 2014a. An automatic and threshold-free performance evaluation system for building extraction techniques from airborne LIDAR data. IEEE Journal of Selected Topics in Applied Earth Observations and Remote Sensing 7(10), pp. 4184-4198.
Awrangjeb, M. and Fraser, C. S., 2014b. Automatic segmentation of raw LIDAR data for extraction of building roofs. Remote Sensing 6(5), pp. 3716-3751.

Champion, N., Boldo, D., Pierrot-Deseilligny, M. and Stamon, G., 2010. 2d building change detection from high resolution satelliteimagery: A two-step hierarchical method based on 3d invariant primitives. Pattern Recognition Letters 31(10), pp. 11381147.

Champion, N., Rottensteiner, F., Matikainen, L., Liang, X., Hyyppa, J. and Olsen, B., 2009. A test of automatic building change detection approaches. International Archives of the Photogrammetry, Remote Sensing and Spatial Information Sciences XXXVIII(3), pp. 49-54.

Grigillo, D., Fras, M. K. and Petrovic, D., 2011. Automatic extraction and building change detection from digital surface model and multispectral orthophoto. Geodetski vestnik 55(1), pp. 2845 .

Ioannidis, C., Psaltis, C. and Potsiou, C., 2009. Towards a strategy for control of suburban informal buildings through automatic change detection. Computers, Environment and Urban Systems 333(1), pp. 64-74.

Matikainen, L., Hyyppa, J., Ahokas, E., Markelin, L. and Kaartinen, H., 2010. Automatic detection of buildings and changes in buildings for updating of maps. Remote Sensing 2(5), pp. 12171248 .

Murakami, H., Nakagawa, K., Hasegawa, H., Shibata, T. and Iwanami, E., 1999. Change detection of buildings using an airborne laser scanner. ISPRS Journal of Photogrammetry and Remote Sensing 54(2-3), pp. 148-152.

Rottensteiner, F., 2007. Building change detection from digital surface models and multispectral images. International Archives of the Photogrammetry, Remote Sensing and Spatial Information Sciences XXXVI(3/W49B), pp. 145-150.

Vosselman, G., Kessels, P. and Gorte, B., 2005. The utilisation of airborne laser scanning for mapping. International Journal of Applied Earth Observation and Geoinformation 6(3-4), pp. 177186.

Zhang, K., Yan, J. and Chen, S. C., 2006. Automatic construction of building footprints from airborne lidar data. IEEE Trans. on Geoscience and Remote Sensing 44(9), pp. 2523-2533.

Zong, K., Sowmya, A. and Trinder, J., 2013. Kernel partial least squares based hierarchical building change detection using high resolution aerial images and lidar data. In: Proc. Digital Image Computing: Techniques and Applications, Hobart, Australia, pp. 1-7. 